\title{
Risk of congenital anomalies in children of parents occupationally exposed to low level ionising radiation
}

Lois M Green, Linda Dodds, Anthony B Miller, Darrell J Tomkins, Jiehui Li, Michael Escobar

Health Services Department, Ontario Hydro, 700 University Avenue, Toronto, Ontario, Canada M5G 1 X6

L M Green

Reproductive Care Program, IWA Grace Health Centre, 5980 University Avenue, Halifax, Nova Scotia, Canada B3H 4N1

L Dodds

Department of Preventive Medicine and Biostatistics, University of Toronto, Toronto, Ontario, Canada M5S 1A8 A B Miller

$\mathrm{J} \mathrm{Li}$

M Escobar

Department of Pediatrics, McMaster University, 1200 Main Street West, Hamilton, Ontario, Canada,

L8N 375

D J Tomkins

Correspondence to: Dr L Green, Health Services Department, Ontario Hydro, 700 University Avenue, Toronto, Ontario, Canada M5G 1 X6.

\begin{abstract}
Objectives-To evaluate the risk of having a child with a congenital anomaly in relation to occupational exposure to low level ionising radiation in the pre-conception period.

Methods-A case-control study based on the Canadian congenital anomalies registry used record linkage techniques to identify congenital anomalies among male and female workers in Canada's largest electric company. Cases were defined as parents of a child with a congenital anomaly born between April 1979 and December 1986 who had a congenital anomaly diagnosed within the first year of life. Controls were an individually matched sample of parents of a liveborn child without an anomaly. Risk of congenital anomaly was assessed in relation to parental exposure to ionising radiation acquired through work within a nuclear generating station of an electric power company. Exposure was assessed according to employment, whether or not the worker was monitored for radiation exposure, and quantitative estimates of radiation dose.
\end{abstract}

Results-Employment within the electric power industry was not associated with an increased risk of congenital anomalies in the offspring of mothers or fathers. Risk estimates for workers monitored (those who are likely to be exposed to ionising radiation) were 1.75 (95\% confidence interval (95\% CI) 0.86 to 3.55$)$ for mothers and $0.84(95 \% \mathrm{CI} 0.68$ to 1.05$)$ for fathers. Exposure for fathers before conception, defined cumulatively and for six months before conception, was not associated with increased risk of anomalies in their offspring. There were no significant increases in risk found between type of anomaly and any measure of exposure, although the statistical power in these groups was limited. The study had insufficient numbers to evaluate the effects of ionising radiation in mothers as only three mothers had recorded doses $>0 \mathrm{mSv}$.

Conclusions-Overall, workers in a nuclear power industry, and specifically those exposed before conception to low levels of ionising radiation, do not appear to be at an increased risk of having a liveborn child with a congenital anomaly.

\section{(Occup Environ Med 1997;54:629-635)}

Keywords: congenital anomalies; ionising radiation; parental occupation; preconceptional exposure

The cause of most congenital anomalies is unknown and their pathogenesis is not well understood. ${ }^{1}$ Those factors which have been established as causing congenital anomalies include: single gene disorders, chromosomal aberrations, multifactorial causes, and discrete environmental teratogens. ${ }^{2}$ Occupational or environmental exposures might be implicated in any of these causal roles.

The possibility that hazardous exposures which parents may receive at work could cause adverse reproductive outcomes is a topic of growing interest. Increasingly there are demands on the part of both the public and workers to know whether reproductive risks exist and to assess their impact. Concerted efforts are being made in many countries to ensure that occupational health policies for workers consider possible risks for their offspring. $^{3}$

Although earlier research was primarily limited to prenatal exposures in the mother, there has been increasing focus in the epidemiological literature on exposures received in the period before conception, and especially to those of the father. ${ }^{4-9}$ Evidence from laboratory studies indicates that defects in offspring can arise from mutagenic exposure of the father. ${ }^{1011}$ Paternal contributions to birth defects can occur as a result of direct gene mutation and chromosomal mutation, or through alterations in the seminal fluid transmitted to the mother. ${ }^{12}$ It is known that radiation has the potential to adversely affect cells, particularly the DNA. ${ }^{13}$ Damage to the DNA is more likely to occur during periods of meiotic division than during periods of inactivity. It has therefore been suggested that men may be at higher risk than women for germ cell damage from radiation as spermatogonia undergo multiple mitotic divisions from the time of sexual maturity. ${ }^{14}$

Studies evaluating the effects of exposures to ionising radiation and reproductive outcomes in humans, specifically congenital anomalies, are very limited. Congenital anomalies considered together are an indication of underlying genetic defects. About half of congenital anomalies in a population based registry were found to have a genetic aetiology. ${ }^{15}$ For the past 
50 years, considerable effort has been made to identify genetic effects of the atomic bombs by studying chromosomal abnormalities, germinal mutations altering protein electrophoresis, and more recently germinal mutations detected at the DNA level. ${ }^{16}{ }^{17}$ These studies have failed to show an increase in germinal mutations in atomic bomb survivors. ${ }^{18}$

However, it has been reported that children born to atomic bomb survivors, conceived on average about five years after the bombings, had an increased, but not significant, risk of a major congenital malformation, stillbirth, and neonatal death with increasing levels of parental exposure to ionising radiation. ${ }^{19}$

Recently an increased germinal mutation rate was found at the DNA level in families exposed to caesium-137 surface contamination after the Chernobyl nuclear power station accident. ${ }^{20}$ Although differences between the types of radiation exposure from the atomic bomb and Chernobyl accidents and the appropriateness of the selected group for comparison may account for the conflicting results, such data suggest a reconsideration of the genetic effects of ionising radiation. ${ }^{21}$

On the other hand, little information is available for chronic low level exposures, which are more representative of contemporary exposures. Few studies have used direct quantitative measures of exposure to radiation. Sever et $a l^{22}$ examined the risk of congenital malformations and exposure to radiation before conception among employees in a plutonium and electrical energy production plant. Although no association was found between maternal exposure before conception and the different birth defects, paternal exposure before conception was significantly related to risk of neural tube defects.

An increased prevalence at birth of central nervous system defects and Down syndrome was found to be associated with the highest concentrations of airborne tritium released in the vicinity of one nuclear plant in southern Ontario. ${ }^{23}$ Inconsistencies related to the exposure data, testing for multiple hypotheses, and the ecological design of the study were cited by the authors as reasons for cautious interpretation of the findings. ${ }^{23}$ The possibility of an association between chromosomal nondisjunction and maternal radiation before conception has been studied for decades with over a dozen studies showing a positive association but most failing to reach significance. ${ }^{24}$

The present case-control study was carried out to evaluate the risk of congenital anomalies in the offspring of parents employed within an electric power company (Ontario Hydro). This company has been responsible for nuclear power generation for the province of Ontario since 1962. Risk of congenital anomalies was assessed from radiation exposure derived from dosimetric measurements and surrogates of this exposure. The case-control design was used as this enabled the same information to be assessed as in a cohort design, but with greater economy of effort.

\section{Methods}

Cases and controls were identified as part of a previously completed study by Dodds et al. ${ }^{25}$ The figure shows the data sources for this study. Cases were ascertained from the Canadian congenital anomalies surveillance system. This is a population based registry of all congenital anomalies diagnosed during the first year of life. In Ontario, the congenital anomaly diagnoses were based on discharge reports from hospital admissions, reports of birth, and death certificates for children under one year of age. The selection of case parents was according to the criteria: child born in Ontario between 1 April 1979 and 31 December 1986, mother resident in Ontario at time of birth of the child, child was liveborn, child had at least one diagnosis with international classification of diseases (ICD-9) congenital anomalies rubrics 740.0-759.9. ${ }^{26} \mathrm{~A}$ maximum of 15 separate diagnoses of congenital anomalies are recorded for each child. One control parent per case was randomly selected from Ontario birth registrations and matched according to the criteria: year of birth of the child, birth order of the child (first, second to third, and fourth or greater), exact maternal age (according to birth year), marital status of mother (single versus not single), and birthplace of each parent (born in Ontario versus not born in Ontario). Parents who were resident outside of Ontario at the time of the index birth and adoptive parents were excluded. To avoid the problem of correlated exposure information among the subjects, parents were only included once even if they had more than one child during the study period. Information from the first birth which occurred during the study period was used, regardless of whether a case or control.

A total of 45200 case-control sets were identified for the mothers and 41158 casecontrol sets were identified for the fathers. There are about $9 \%$ fewer fathers than mothers because fathers are not always named on the birth certificates-for example, in the case of a single mother.

The case and control parents were then linked with a stepwise set of computer linkages based on a probabilistic linking model ${ }^{27}{ }^{28}$ with a cohort of 32975 men and 9507 women who worked for Ontario Hydro. This cohort represented employees with at least 12 consecutive months of employment at Ontario Hydro during the period 1978-86 inclusive. The inclusion criterion of 12 consecutive months of employment was imposed because the company did not require complete retention of records for employees who worked less than 12 months. As the accuracy and completeness of the record linkage process depended on personal identifiers being present, the group where these identifiers might be incomplete was excluded. To resolve any uncertainties on possible matches between the case-control sets and the employee cohort, additional personal identifiers were obtained from company records.

For men, the period of aetiological interest is before conception. The date of conception was determined by subtracting gestational age from 
the date of birth for the child of the case or control parent. A comparison was made between date of hire with the company and date of conception. If the date of conception preceded the date of employment, these parents were excluded. For women, the periods of interest included employment before conception as well as employment during pregnancy.

In accordance with legislative requirements for regulatory control, ${ }^{29}$ all workers whose exposures to ionising radiation may exceed the dose limits for the members of the general public are monitored and dose records are retained. Radiation doses acquired before employment with Ontario Hydro are collected and are added to those doses received during employment with Ontario Hydro. ${ }^{30}$ Doses were estimated by methods and measuring devices used for personal monitoring calibrated in accordance with the specifications of the Atomic Energy Control Board of Canada.

Detailed dose information is kept for external whole body dose (which includes any neutron dose), external skin dose, and internal dose (primarily from uptakes of tritium) which are the most common exposures associated with work in the nuclear power generation sector. For the years 1962-76, radiation doses from $x$ rays, $\gamma$ rays, and $\beta$ rays were monitored with a film badge. From 1976 to the present, doses of $x$ rays, $\gamma$ rays, and $\beta$ rays were monitored with a thermoluminescent dosimeter. Internal dose, reflecting the internal uptake of radionuclides, mainly tritium, was measured with urinary bioassay.

The risk of congenital anomalies was determined for three main types of exposure: employment with Ontario Hydro, monitoring status (those employees monitored in accordance with regulatory requirements), and quan- titative estimates for radiation dose. For the quantitative estimates, three periods of aetiological interest were evaluated: total whole body dose before conception, whole body dose six months before conception, and tritium dose 60 days before conception. The six month period allowed comparability with the study of Gardner $e t a^{\beta 1}$ in which an excess risk of childhood cancer was found among offspring of parents occupationally exposed to ionising radiation and which was more pronounced in the period six months before conception. The 60 day period corresponded with the period of human spermatogenesis. ${ }^{32}$

Congenital anomalies may be multiple in expression and may have diverse aetiologies. As many as 15 anomalies per child coded to ICD-9 were recorded. A child with more than one anomaly was counted in more than one ICD group in the analyses according to ICD category.

To categorise anomalies according to their possible aetiology, a hierarchical system developed by Baird $e t a l^{15}$ was used to establish aetiological groupings. The assignment of aetiological groupings based on ICD codes was carried out by a geneticist (DJT) who was not aware of the employment or exposure status of the parent. Each child was assigned to one of the aetiological groups in the following hierarchy: single gene disorders (autosomal dominant, autosomal recessive, $\mathrm{X}$ linked), chromosomal (autosomal and $\mathrm{X}$ chromosomal), multifactorial, genetic (unspecified), and unknown. This hierarchical system ensured that cases were not counted more than once and also permitted comparisons with other work. ${ }^{15} 25$

Because the mechanisms by which ionising radiation might affect the risk of congenital anomalies is different for offspring of men and

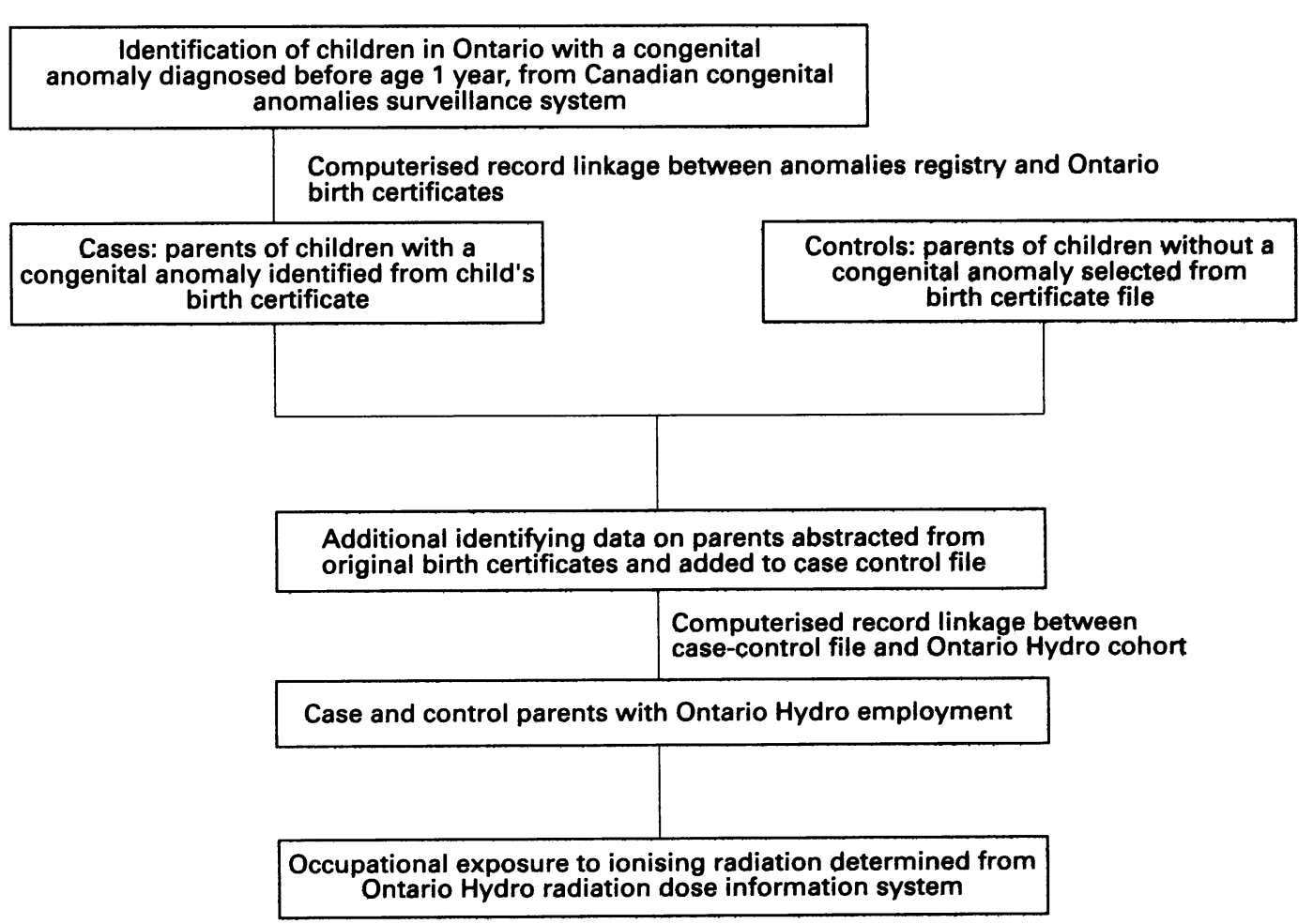


Table 1 Exposure information for fathers * according to period before conception

\begin{tabular}{lll}
\hline Exposure & Cases & Controls \\
\hline Employed but not monitored for radiation exposure & 189 & 247 \\
Monitored for radiation exposure, recorded dose $=0 \dagger$ & 63 & 53 \\
Monitored for radiation exposure, recorded dose $>0$ (mSv)t: & & \\
Cumulative whole body dose before conception: & 89 & 126 \\
Number of employees & $39.9(64.4)$ & $38.7(58.9)$ \\
Mean (SD) dose & $0.04-262.1$ & $0.10-307.0$ \\
Dose range & & 73 \\
Whole body dose six months before conception: & 65 & $3.6(4.1)$ \\
Number of employees & $3.6(4.5)$ & $0.01-17.4$ \\
Mean (SD) dose & $0.05-18.2$ & \\
Dose range & & 97 \\
Cumulative tritium dose before conception: & 74 & $14.4(23.9)$ \\
Number of employees & $16.9(27.2)$ & $0.03-137.9$ \\
Mean (SD) dose & $0.02-107.2$ & \\
Dose range & & 53 \\
Tritium dose received 60 days before conception: & 51 & $0.5(0.6)$ \\
Number of employees & $0.4(0.5)$ & $0.02-2.8$ \\
Mean (SD) dose & $0.01-2.8$ & \\
Dose range & & \\
\hline
\end{tabular}

*Only three mothers had a non-zero dose.

†Dose refers to that received at Ontario Hydro from date of hire to date of conception including any dose reported at the time of hire by Ontario Hydro.

women, fathers and mothers were analysed separately. The closeness of the matching together with the type of records (birth registrations) from which the data for this study were gathered limited the number of variables available for evaluation as possible risk factors or confounders. Other than the matching variables, history of stillbirth ${ }^{33}$ and paternal age a $^{34}$ were the only available variables which might be predictive of risk of congenital anomaly. As the data were collected with a matched case-control design, the standard McNemar test $^{35}$ and conditional logistic regression ${ }^{36}$ were used for the analysis. Maximum likelihood estimates for odds ratios (ORs) were calculated with SAS for matched pairs that were discordant for the exposure of interest in the parent. The method of Miettinen was used to calculate the $95 \%$ confidence interval $(95 \% \mathrm{CI}){ }^{37}$

\section{Results}

A total of 763 pairs of case-control fathers and 165 pairs of case-control mothers were identified in which at least one parent had employment at Ontario Hydro. For fathers, four pairs were concordant for employment with Ontario Hydro-for example, both case and control had been employed with Ontario Hydro. Analysis of all anomalies combined showed no evidence of increased risk in relation to employment of the parents at Ontario Hydro (for men, OR $0.79,95 \%$ CI 0.69 to 0.92 ; for women, OR $0.94,95 \%$ CI 0.69 to 1.28 ).

As a surrogate of exposure to ionising radiation, monitoring status was examined in relation to congenital anomalies in the offspring. A monitored worker may be expected to have exposure to ionising radiation greater than that which would normally be experienced by members of the general public. A monitored worker is not necessarily synonymous with an exposed worker. There were 149 pairs where the case father was monitored for exposure to radiation and the control father was not and 177 pairs where the case father was not monitored and the control father was. The analysis of these discordant pairs gave an OR of 0.84 ( 0.68 to 1.05 ). For mothers, the corresponding OR was 1.75 (0.85 to 3.55$)$ which was based on substantially fewer discordant pairs, 21 pairs where the case mother was monitored and the control mother was not and 12 pairs where the case mother was not monitored and the control mother was.

For fathers, more detailed analyses were carried out according to radiation dose and period before conception. Table 1 shows exposure information relating to dose and period before conception. Of the 331 workers who were monitored for exposure to radiation, $35 \%$ had a recorded dose of zero $\mathrm{mSv}$. The categories of zero dose and no exposure opportunity were combined to form the control exposure variable in the conditional logistic regression models. Regulations in effect during the study period were such that few women worked in areas where radiation exposure would be expected. It was not possible to conduct this level of analysis for the mothers because only three mothers (two cases, one control) had recorded radiation exposure $>0 \mathrm{mSv}$.

Table 2 shows adjusted ORs, according to aetiological subgroups, and father's whole body dose, and tritium dose for specified periods before conception. With the exception of the risk estimate for anomalies classified as having a multifactorial aetiology, which did attain significance for cumulative exposure only (OR $0.61,95 \%$ CI 0.42 to 0.90 ), the ORs for specific aetiological groups were not significantly different from unity. Risk estimates for chromosomal anomalies were above unity, but wide CIs indicate the instability of the estimates which are based on few discordant pairs.

Adjusted ORs, ICD groupings, father's whole body dose, and tritium dose (table 3 ), were also not significantly increased or reduced, with the exception of anomalies of the circulatory system which showed a significantly reduced OR for cumulative exposure only $(0.51,95 \%$ CI 0.27 to 0.95$)$ and musculoskeletal anomalies which showed a reduced OR of borderline significance $(0.62,95 \%$ CI 0.38 to 1.01). Odds ratios above unity (not significant) were found for chromosomal anomalies for fathers who had a recorded dose greater than zero either six months or 60 days before 
Table 2 Adjusted ORs (95\% CIs) for aetiological groups of congenital anomalies according to dose ${ }^{\star}$ and radiation exposure of fathers during the period before conception

\begin{tabular}{|c|c|c|c|c|c|c|}
\hline \multirow[b]{2}{*}{ Aetiological group } & \multicolumn{2}{|c|}{$\begin{array}{l}\text { Cumulative whole body dose before } \\
\text { conception }\end{array}$} & \multicolumn{2}{|c|}{ Whole body dose 6 months before conception } & \multicolumn{2}{|c|}{ Tritium dose 60 days before conception } \\
\hline & Discordant pairst & $O R(95 \% C I)$ & Discordant pairs & OR $(95 \% C I)$ & Discordant pairs & OR $(95 \% C I)$ \\
\hline Single gene disorders $\ddagger$ & $0 / 3$ & - & $0 / 0$ & - & $0 / 0$ & - \\
\hline Chromosomal disorders $\ddagger$ & $4 / 4$ & $0.97(0.24$ to 3.91$)$ & $3 / 2$ & $1.46(0.24$ to 8.80$)$ & $3 / 2$ & $1.46(0.24$ to 8.80$)$ \\
\hline Multifactorial\$ & $43 / 70$ & $0.61(0.42$ to 0.90$)$ & $31 / 37$ & $0.92(0.56$ to 1.50$)$ & $28 / 28$ & $1.13(0.66$ to 1.94$)$ \\
\hline Genetic, unspecified $\ddagger$ & $13 / 14$ & $0.93(0.44$ to 1.98$)$ & $8 / 10$ & $0.83(0.33$ to 2.12$)$ & $6 / 8$ & $0.80(0.27$ to 2.32$)$ \\
\hline Unknown』 & $28 / 34$ & $0.80(0.48$ to 1.32$)$ & $22 / 23$ & $0.91(0.50$ to 1.65$)$ & $14 / 15$ & $0.84(0.40$ to 1.76$)$ \\
\hline Totals & $88 / 125$ & $0.72(0.55$ to 0.95$)$ & $64 / 72$ & $0.90(0.60$ to 1.27$)$ & $51 / 53$ & $0.99(0.67$ to 1.47$)$ \\
\hline
\end{tabular}

$\star$ Dose $>0 v$ dose $=0$ or not exposed.

†Case exposed, control not exposed, or case not exposed, control exposed.

$¥$ Adjusted for father's age only.

¿Adjusted for father's age and history of stillbirths.

- Could not be calculated.

conception, but the estimates were based on small numbers with wide $95 \%$ CIs (table 3 ). There was a tendency in several ICD groups of anomalies for the ORs to increase for the period closest to the date of conception but all were accompanied by wide $95 \%$ CIs, of which none were significant at the $5 \%$ level.

\section{Discussion}

We found no evidence of a significantly increased risk for fathers having a liveborn child with a congenital anomaly after occupational exposure to radiation in the period before conception. Congenital anomalies do not all share the same aetiological factors and combining all anomalies together may dilute the risk that ionising radiation may have on some specific anomalies. However, analyses according to subgroups, defined by possible aetiology or by anatomical ICD rubrics, also showed no evidence that fathers with exposure to ionising radiation were more likely to have a child with a birth defect. There is not sufficient evidence from this study to suggest whether this relation is causal or not. Further studies with larger numbers are required to confirm or rule out this relation.

For mothers, the opportunities for exposure during the study period were minimal as dictated by regulatory requirements, and hence, the data for mothers were limited. The available data indicated, as was shown for fathers, no significantly increased risks of having a child with a congenital anomaly relative to employment or monitoring status. The non- significant increase in risk for mothers who were monitored might be explained by chance alone. In this study we did not have the power to fully explore the relation between exposure to ionising radiation in mothers and congenital anomalies in their offspring, but the suggestion of an increased risk among those who were monitored may warrant further study.

Calculations carried out at the end of the work $^{38}$ indicated a statistical power of over $80 \%$ to detect a 1.5 increase in risk for all anomalies combined among fathers relative to their exposure before conception. However, the statistical power was greatly reduced in the analyses for mothers and by aetiological subgroups for offspring of fathers and hence a true association might have been missed because of this.

The design of this study provided distinct strengths in the evaluation of risks of congenital anomaly potentially associated with exposure to ionising radiation. There is no known reporting bias in the population based registry from which the cases were ascertained. The acquisition of dosimetric measurements of exposure to radiation with record linkage techniques and no direct contact with study participants, obviated any possibility of recall bias, which can compromise the validity of studies examining a health outcome of relatively high emotion and an earnest desire to understand the cause.

The measurement of exposure to ionising radiation was blind to case-control status and thereby avoided any possible bias in exposure assessment. Quantitative dose estimates were

Table 3 Adjusted ORs (95\% CIs) for ICD groupings of congenital anomalies according to dose* and radiation exposure of fathers during the period before conception

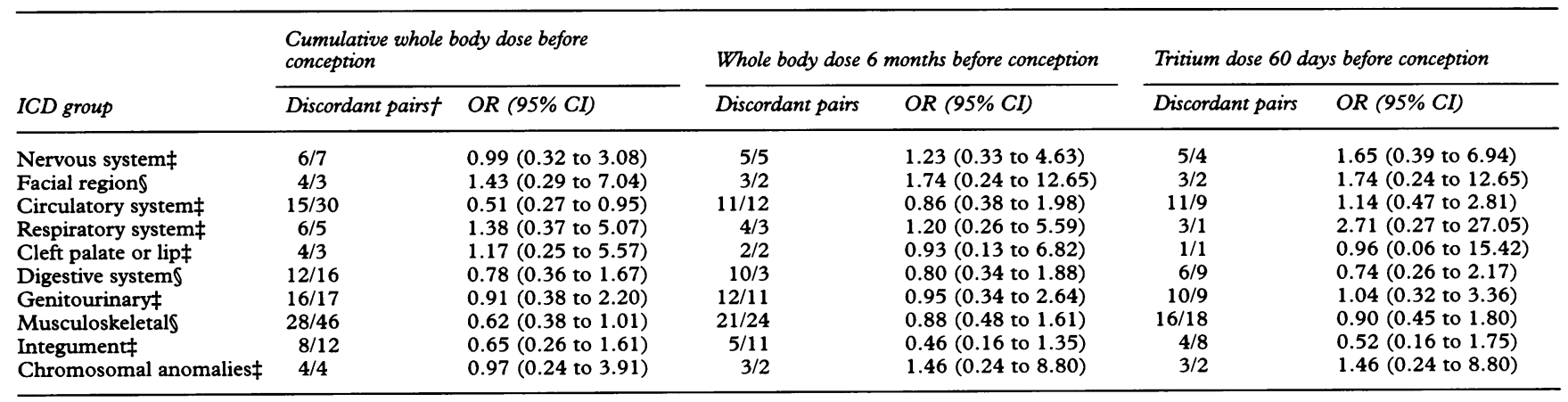

«Dose $>0$ v dose $=0$ or not exposed.

tCase exposed, control not exposed, or case not exposed, control exposed.

$\ddagger$ Adjusted for father's age.

SAdjusted for father's age and history of stillbirths. 
assigned in advance of the outcome occurrence, having been previously documented in accordance with independently determined regulatory guidelines and controls which establish standards of accuracy.

In this study, it was expected that availability of personal identifiers from employment records would contribute to the accuracy of case and control ascertainment through the population based records as record linkage techniques were used. Whether this accuracy has indeed been optimised might be questioned in the light of several estimates of risk which were below unity. There was, at the start of the study, no reason why exposure to ionising radiation should be associated with a reduced risk of anomalies, unless this exposure selectively decreased fertility (reduced ability to conceive among parents who would have been more likely to have a fetus with a congenital anomaly or increased spontaneous abortions or stillbirths among fetuses with an anomaly). Therefore, it is possible that the stringent criteria used for linkages might have contributed to an underascertainment of a few cases although it would not likely be differential to case-control status.

Limitations of the study relate to incomplete control for possible confounders. However, the closeness of the matching in the study design did control for several possible confounders. Information relating to medical history of the parents was not available and therefore, we were unable to account for the possible role of maternal illnesses before and during pregnancy, relevant family medical history, reproductive history, or socioeconomic status. The analyses pertaining to aetiological subgroups were further limited by the availability of only four digit ICD-9 codes making it difficult to assign aetiology with certainty. For this reason, many anomalies had unknown aetiology or unspecified genetic aetiology. However, in studies of congenital anomalies, it is important to group together anomalies with common aetiology. Seldom will studies be large enough to have the power to analyse any single anomaly.

This study is confined to those congenital anomalies in liveborn infants resulting from "successful conceptions" and pregnancies and therefore, is restricted to a subset of fertile mothers and fathers. It was not possible for this study to consider if ionising radiation compromises fertility in any way. The lack of an effect in liveborn children may be the result of increased effects of other adverse reproductive outcomes. However, because the selection of controls took parity into account, and by doing so provided some control for fertility, it is unlikely that this factor constitutes a major limitation.

The epidemiological evidence to which these findings could be related is sparse. The findings reported here do not correspond with those reported by Sever $e t a l,{ }^{22}$ one of the few studies that used quantitative individual measurements of exposure to radiation. They found an increase in neural tube defects related to exposure of fathers to ionising radiation before con- ception. The ecological design of the study by Johnson and Rouleau, ${ }^{23}$ who found an increase in Down's syndrome relative to tritium releases from nuclear power generating stations causes difficulties in interpretation of the results at the individual level, and in relating the findings to those from studies such as this one.

The present study is unique in its examination of the possible role of exposure to ionising radiation before conception as we used existing individual dosimetric exposure records and a population based birth defects registry. Although workers involved in the generation of nuclear power receive higher exposures of ionising radiation than environmental exposures which might be received by members of the public, the absence of a significantly increased risk of having a child with a congenital anomaly in fathers exposed to radiation within an occupational setting suggests that a public health risk is unlikely.

We acknowledge Claus Wall who performed data management activities and record linkage and Warren Christiani who provided the exposure information related to ionising radiation. This study was supported by Grant 04450 Ontario, Ministry of Health, and by Ontario Hydro.

1 Kalter J, Warkany J. Congenital malformations: etiologic factors and their role in prevention. $N$ Engl $\mathcal{F}$ Med 1983;308:424-31.

2 Baird PA. Reproductive hazards and the workplace [editorial]. Can Med Assoc F 1992;147:157-60.

3 Stijkel A, van Dijk FJH. Developments in reproductive risk management. Occup Environ Med 1995;52:294-303.

4 Taskinen HK. Effects of parental occupational exposures on Taskinen HK. Effects of parental occupational exposures on spontaneous abortions and congenital ma

5 Roman E, Doyle P, Ansell P, Bull D, Beral V. Health of children born to medical radiographers. Occup Environ Med 1996;53:73-9.

6 Brender JD, Suarez L. Paternal occupation and anencephaly. Am f Epidemiol 1990;131:517-21

7 McDonald AD, McDonald JC, Armstrong B, Cherry NM Nolin AD, Robert D. Fathers' occupation and pregnanc outcome. Br F Ind Med 1989;46:329-33.

8 Olshan A, Teschke K, Baird PA. Paternal occupation and congenital anomalies in offspring. Am f Ind Med 1991;20: 447-75.

9 Olshan AF, Baird PA, Teschke K. Paternal occupational exposures and the risk of Down's syndrome. Am $₹$ Hum Genet 1989;44:646-51.

10 Nomura T. Parental exposure to x-rays and chemicals induces heritable tumours and anomalies in mice. Nature 1982;296:755-7.

11 Kirk KM, Lyon MF. Induction of congenital malformations in the offspring of male mice treated with $x$-rays at pre-meiotic and post-meiotic stages. Mutat Res 1984;125 75-85.

12 Olshan AF, Faustman E. Male-mediated developmental toxicity. Annu Rev Public Health 1993;14:159-81.

13 Parker RG. Principles of radiation oncology. In: Haskell CM, ed. Cancer treatment. Philadelphia: WB Saunders, 1985, 14-20.

14 Connor JM, Ferguson-Smith MA. Essential medical genetics. Oxford: Blackwell, 1987.

15 Baird PA, Anderson TW, Newcombe HB, Lowry RB. Genetic disorders in children and young adults: Genetic disorders in children and young adults:

16 Kodaira M, Satoh C, Hiyama K, Toyama K. Lack of effects of atomic bomb radiation on genetic instability of tandemrepetitive elements in human germ cells. Am $\mathcal{F}$ Hum Gene 1995;57:1275-83.

17 Neel JV. New approaches to evaluating the genetic effects of the atomic bombs. Am f Hum Genet 1995;57:1263-6.

18 Sankaranarayanan $K$. Ionizing radiation, genetic risk estimation and molecular biology: impact and inferences. Trends Genet 1993;9:79-84.

19 Otake M, Schull WJ, Neel JV. Congenital malformations, stillbirths, and early mortality among the children of atomic bomb survivors: a reanalysis. Rad Res 1990;122:111.

20 Dubrova YE, Nesterov VN, Krouchinsky NG, Ostapenki VA, Neumann R, Nell DL, Jeffreys AJ. Human minisatellite mutation rate after the Chernobyl accident. Nature 1996;380:683-6.

21 Satoh C, Kodaira M. Effects of radiation on children. Nature 1996;383:226.

22 Sever LE, Gilbert ES, Hessol NA, McIntyre JM. A case-control study of congenital malformations and occupational exposure to low-level ionizing radiation. Am $f$ Epipational exposure to low-ley 
23 Johnson KC, Rouleau J. Tritium releases from the Pickering nuclear generating station and birth defects and infant mortality in nearby communities, 1971-88. Ottawa, Canada: Atomic Energy Control Board, 1991. (AECB Project No 7156.1.)

24 Uchida IA. Radiation-induced non-disjunction. Environ Health Perspect 1979;31:13-7.

25 Dodds L, Marrett LD, Tomkins DJ, Green B, Sherman G. Case-control study of congenital anomalies in children of Case-control study of congenital anomatie

26 World Health Organization. The international classification of diseases, 9th revision. Vol 1. Geneva: World Health diseases, 9th

27 Roos LL, Wajda A, Nicol JP. The art and science of the record linkage: methods that work with few identifiers. Comput Biol Med 1986;16:45-57.

28 Roos LL, Sharp SM, Nichol JP. Software for health care analysts; a modular approach. F Med Syst 1987;11:445-64.

29 Atomic Energy Control Act. Atomic Energy Control Board Regulations fune 1974. Ottawa, Canada: Minister of Supply and Services, 1974. (Registration SOR/74-334.)

30 Nuclear Generation Division Radioactivity Management and Environmental Protection. Radioactivity Management and Ontario Hydro, 1980.
31 Gardner MJ, Snee MP, Hall CA, Downes S, Terrell JD. Results of a case-control study of leukaemia and lymphoma among young people near Sellafield nuclear plant in West Cumbria. BMF 1990;300:423-9.

32 Courot $M$, Hochereau-de Reviers MT, Ortavant $R$. Spermatogenesis: In: Johnson AD, Gomes WR, Vandemark NL, eds. The testis. New York: Academic Press, 1970.

33 Lie RT, Wilcox AJ, Skjaerven R. A population-based study of the risk of recurrence of birth defects. N Engl $\mathcal{F}$ Med 1994;331:1-4.

34 McIntosh GC, Olshan AF, Baird PA. Paternal age and the risk of birth defects in offspring. Epidemiology 1995;6:282-8. 35 Fleiss J. Statistical methods for rates and proportions. New York: Wiley, 1981.

36 Hosmer DW, Lemeshow S. Applied logistic regression. New York: Wiley, 1989.

37 Miettinen OS. Estimation of relative risk from individually matched sets. Biometrics 1970;26:75-86.

38 Schlesselman J. Case control studies: design, conduct and analysis. Oxford: Oxford University Press, 1982.

\section{Vancouver style}

All manuscripts submitted to Occup Environ Med should conform to the uniform requirements for manuscripts submitted to biomedical journals (known as the Vancouver style.)

Occup Environ Med, together with many other international biomedical journals, has agreed to accept articles prepared in accordance with the Vancouver style. The style (described in full in the $\mathcal{F A M A [ 1 ] )}$ is intended to standardise requirements for authors, and is the same as in this issue.

References should be numbered consecutively in the order in which they are first mentioned in the text by Arabic numerals on the line in square brackets on each occasion the reference is cited (Manson[1] confirmed other reports [2][3][4][5]). In future references to papers submitted to Occup Environ Med should include: the names of all authors if there are seven or less or, if there are more, the first six followed by $e t$ al; the title of journal articles or book chapters; the titles of journals abbreviated according to the style of Index Medicus; and the first and final page numbers of the article or chapter. Titles not in Index Medicus should be given in full.

Examples of common forms of references are:

1 International Committee of Medical Journal Editors. Uniform requirements for manuscripts submitted to biomed journals. $\mathcal{F A M A}$ 1993;269:2282-6.

2 Soter NA, Wasserman SI, Austen KF. Cold urticaria release into the circulation of histmaine and eosinophil chemotactic factor of anaphylaxis during cold challenge. N Engl f Med 1976;294:687-90.

3 Weinstein L, Swartz MN. Pathogenic properties of invading micro-organisms. In: Sodeman WA Jr, Sodeman WA, eds. Pathologic physiology, mechanisms of disease. Philadelphia: W B Saunders, 1974:457-72. 